\title{
EVALUACIÓN DE LA POROSIDAD DEL CONCRETO MODIFICADO CON ADICIÓN DE FIBRAS DESHIDRATADAS DE CACTUS
}

\author{
N. DÍAZ ${ }^{1}$, W. MARTÍNEZ ${ }^{2}$, L. A. GONZÁLEZ ${ }^{1}$, J. A. GUZMÁN ${ }^{3}$, M. A. NAVARRETE ${ }^{3}$ \\ ${ }^{1}$ Facultad de Ingeniería Civil, Universidad Michoacana de San Nicolás de Hidalgo, Morelia, Michoacán, México 58040. \\ Gral Francisco J. Múgica Edificio F. \\ ${ }^{2}$ Cuerpo Académico Consolidado-147, Facultad de Ingeniería Civil, Universidad Michoacana de San Nicolás de Hidalgo, \\ Morelia, Michoacán, México 58040. Gral Francisco J. Múgica Edificio C. \\ ${ }^{3}$ Facultad de Ciencias Físico Matemáticas, Universidad Michoacana de San Nicolás de Hidalgo, Morelia, Michoacán, \\ México 58030. Gral Francisco J. Múgica Edificio B.
}

\begin{abstract}
RESUMEN
Este trabajo analiza la porosidad de cuatro mezclas de concreto a una edad de 8 años de su elaboración; mezcla con agregado grueso triturado "TT", mezcla con agregado grueso triturado más un $2 \%$ de adición de cactus en base a la masa del cemento "TTC", mezcla con agregado grueso de canto rodado "TR" y mezcla con agregado grueso de canto rodado más un $2 \%$ de adición de cactus en base a la masa del cemento "TRC". La porosidad se obtuvo mediante el ensayo llamado absorción capilar durante un tiempo de 30 días. Los resultados obtenidos indican que la materia orgánica que se utilizó como adición incrementa la absorción dentro de la probeta, lo cual no es benéfico para el concreto.
\end{abstract}

Palabras clave: concreto; cactus; cemento; pétreos; porosidad.

\begin{abstract}
This work analyzes the porosity of four concrete mixtures at an age of 8 years of its elaboration; mix with crushed coarse aggregate "TT", mix with crushed coarse aggregate plus $2 \%$ addition of cactus based on the mass of the "TTC" cement, mix with coarse aggregate of "TR" pebble and mix with coarse aggregate of edge rolled plus $2 \%$ cactus addition based on the "TRC" cement mass. The porosity was obtained by the test called capillary absorption for a period of 30 days. The results obtained indicate that the organic matter that was used as an addition increases the absorption inside the test tube, which is not beneficial for the concrete.
\end{abstract}

Keywords: concrete; cactus; cement; stony; porosity.

\section{RESUMO}

Este trabalho analisa a porosidade de quatro misturas de concreto aos 8 anos de sua elaboração; misturar com agregado grosso triturado "TT", misturar com agregado grosseiro triturado mais adição de $2 \%$ de cactus com base na massa do cimento "TTC", misturar com agregado graúdo de pedrisco "TR" e misturar com agregado graúdo de borda adição de cactos laminados mais $2 \%$ com base na massa de cimento "TRC". A porosidade foi obtida pelo teste chamado de absorção capilar por um período de 30 dias. Os resultados obtidos indicam que a matéria orgânica utilizada como adição aumenta a absorção dentro do tubo de ensaio, o que não é benéfico para o concreto.

Palavras-chave: concreto; cacto; cimento; pedregoso; porosidade 


\section{INTRODUCCIÓN}

El cemento es uno de los materiales más producido en el mundo y con más empleo en la ingeniería civil, sin embargo, su producción genera alta contaminación, por lo que se buscó incorporar una adición que mejorara las características del concreto y que sea económica, de esta manera se utilizó fibra deshidratada de nopal como adición en el concreto.

Se realizaron probetas prismáticas que fueron probadas a flexión, a las cuales se les extrajeron dos núcleos de concreto, que fueron los analizados por porosidad en este trabajo. Se realizaron pruebas de absorción capilar durante 30 días a 4 tipos de mezclas, obteniéndose resultados interesantes.

\section{PROCEDIMIENTO}

Primeramente se eligieron los materiales a utilizar para el concreto: cemento CPO 30R RS BRA "Cemento Portland Ordinario" Resistente a los sulfatos con Baja Reactividad Álcali Agregado, agua potable de la red pública, aditivo de cactus, agregados pétreos (NMX-C-030-ONNCCE-2004) de origen volcánico, basáltico y de rio, de Morelia Michoacán, a los cuales se les determinaron sus características mecánicas, para el diseño de mezclas ACI (ACI 211.1-91).

A los agregados pétreos se le realizaron pruebas de: \% Humedad Actual (NMX-C-166-ONNCCE2006), \% Humedad de Absorción (NMX-C-165-ONNCCE-2004, NMX-C-164-ONNCCE-2004) Densidad (ASTMC 128-01), Masa volumétrica Seca varillada (NMX-C-073-ONNCCE-2004), Masa volumétrica Seca Suelta (NMX-C-073-ONNCCE-2004), y Granulometría (NMX-C-077-1997ONNCCE). Al cemento utilizado se le realizaron pruebas de: Densidad (NMX-C-152-ONNCCE-2010) y Tiempos de Fraguado (NMX-C-059-1997-ONNCCE), para verificar su reacción. Al agua se le realizaron análisis químicos de acuerdo con la norma (NMX-C-122-ONNCCE-2004), para garantizar su pureza. Al concreto fresco se le hicieron pruebas de revenimiento, para analizar su fluidez de acuerdo con el método ACI (ACI 211.1-91).

Se elaboraron 4 mezclas para un f'c de $250 \mathrm{~kg} / \mathrm{cm}^{2}$ de acuerdo con el diseño ACI, se fabricaron 9 vigas rectangulares de cada mezcla (NMX-C-159-ONNCCE-2004) y después de 24 horas se desmoldaron, para comenzar su curado por inmersión hasta cumplir la edad de prueba de 8 años. Se probaron a flexión y después se extrajeron 2 núcleos de cada viga para realizar la prueba de absorción capilar durante 30 días (RED DURAR), con la finalidad de analizar el efecto de la fibra en el concreto respecto a la porosidad.

\section{RESULTADOS}

En la Tabla 1 se presentan los resultados promedios de las características de los materiales. Obteniéndose los valores de \% de humedad actual, \% de humedad de absorción y densidad de acuerdo con las normas NMX-C-166-ONNCCE-2006, NMX-C-165-ONNCCE-2004, ASTMC 128-01 y NMX-C-083-ONNCCE; PVSS y PVSV de acuerdo con la norma NMX-073-ONNCCE-2004; MF y TM de acuerdo a la norma NMX-077-1997-ONNCCE. 
Tabla 1. Características de los materiales usados.

\begin{tabular}{cccccccc}
\hline \multicolumn{7}{c}{ CARACTERÍSTICAS DE LOS MATERIALES } \\
\hline MATERIAL & $\begin{array}{c}\text { \% H. } \\
\text { ACTUAL }\end{array}$ & $\begin{array}{c}\text { \% H. } \\
\text { ABSORCIÓN }\end{array}$ & $\begin{array}{c}\text { DENSIDAD } \\
\left(\mathbf{g} / \mathbf{c m}^{\mathbf{3}}\right)\end{array}$ & $\begin{array}{c}\text { PVSS } \\
\left(\mathbf{k g} / \mathbf{c m}^{\mathbf{3}}\right)\end{array}$ & $\begin{array}{c}\text { PVSV } \\
\left(\mathbf{k g} / \mathbf{c m}^{\mathbf{3}}\right)\end{array}$ & MF & TM \\
$\begin{array}{c}\text { Arena } \\
\text { volcánica }\end{array}$ & 1.88 & 4.91 & 2.320 & 1205.15 & 1332.00 & 3.39 & \\
$\begin{array}{c}\text { Grava } \\
\text { redondeada } \\
\text { (de rio) }\end{array}$ & 1.42 & 2.05 & 2.830 & 1445.40 & 1527.33 & $3 / 4 "$ \\
$\begin{array}{c}\text { Grava } \\
\text { triturada }\end{array}$ & 1.21 & 2.41 & 2.710 & 1381.60 & 1506.60 & $3 / 4 "$ \\
Cemento & & 3.176 & 1490.00 & & & \\
\hline
\end{tabular}

En la figura 1 se muestra el comportamiento del concreto a la penetración de agua, lo cual depende del tipo de porosidad que presente la muestra, obteniéndose resultados muy similares y ligeramente superiores las mezclas que contienen la adición de cactus.

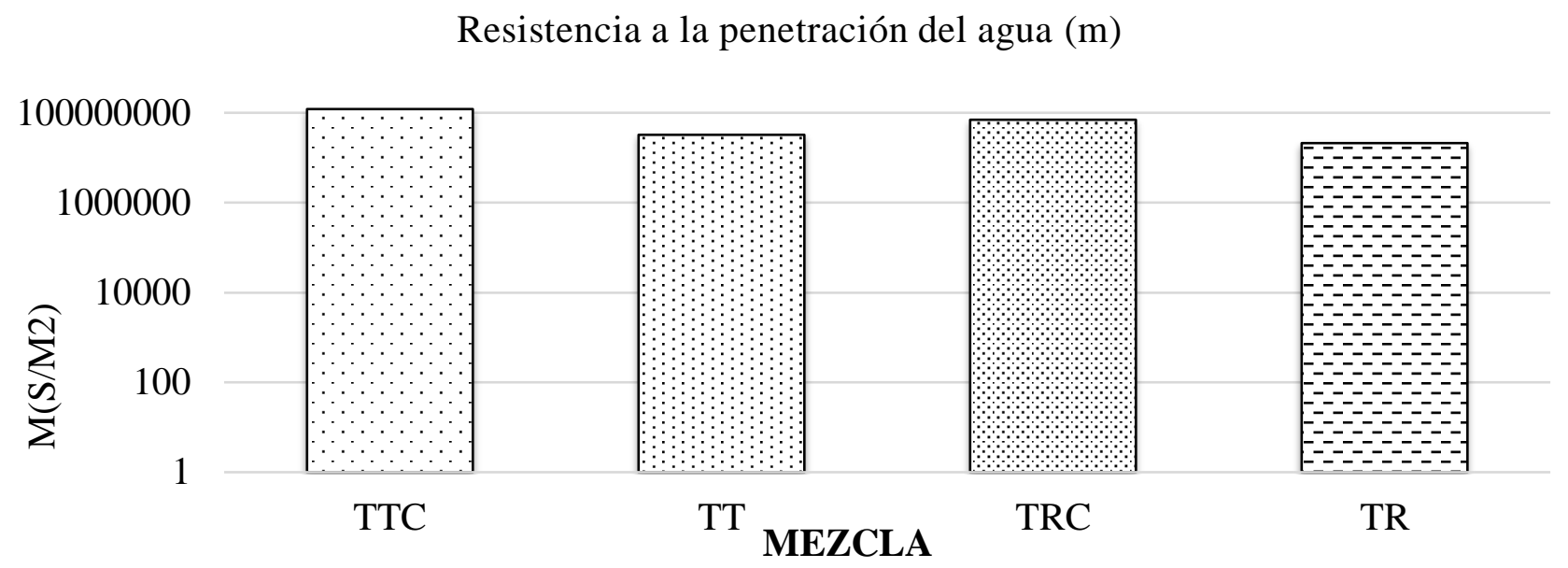

Figura 1. Penetración de agua.

En las figuras 2 se muestra la porosidad efectiva de cada mezcla, obteniéndose mejores resultados en el concreto que con la adición de cactus.

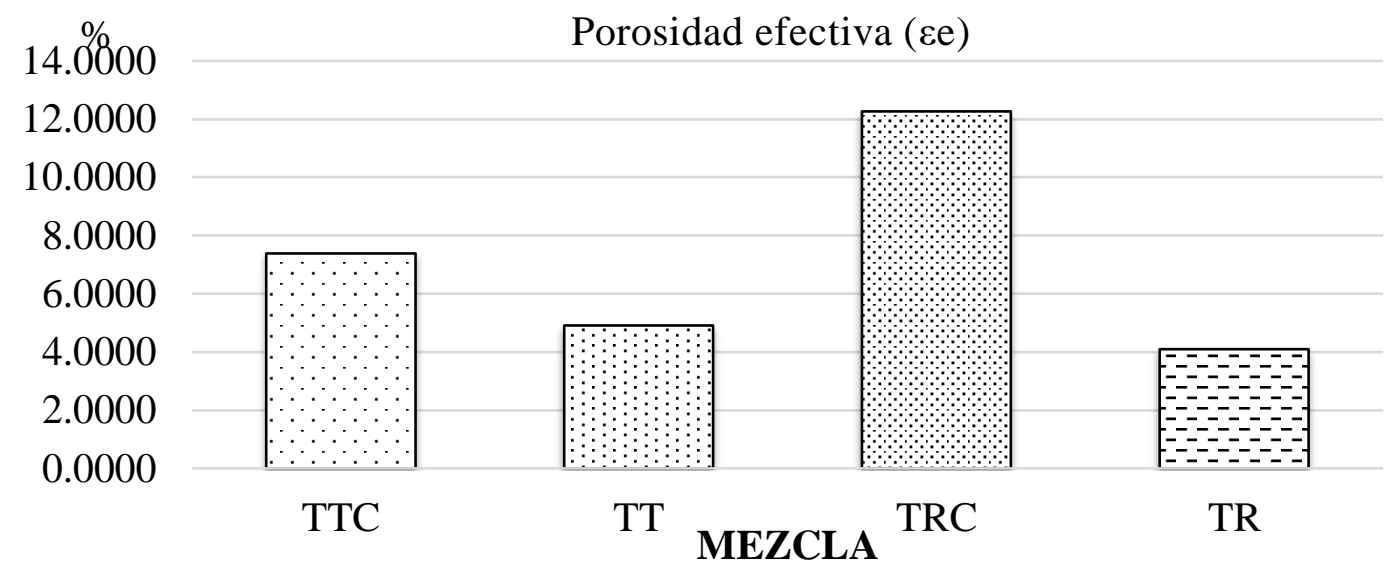

Figura 2. Porosidad efectiva. 
En las figuras 3 se muestran los resultados de absorción capilar, obteniendo un mayor resultado en la mezcla de agregado triturado con la adición de cactus.

\section{Coeficiente de absorción capilar (k)}

0.1000

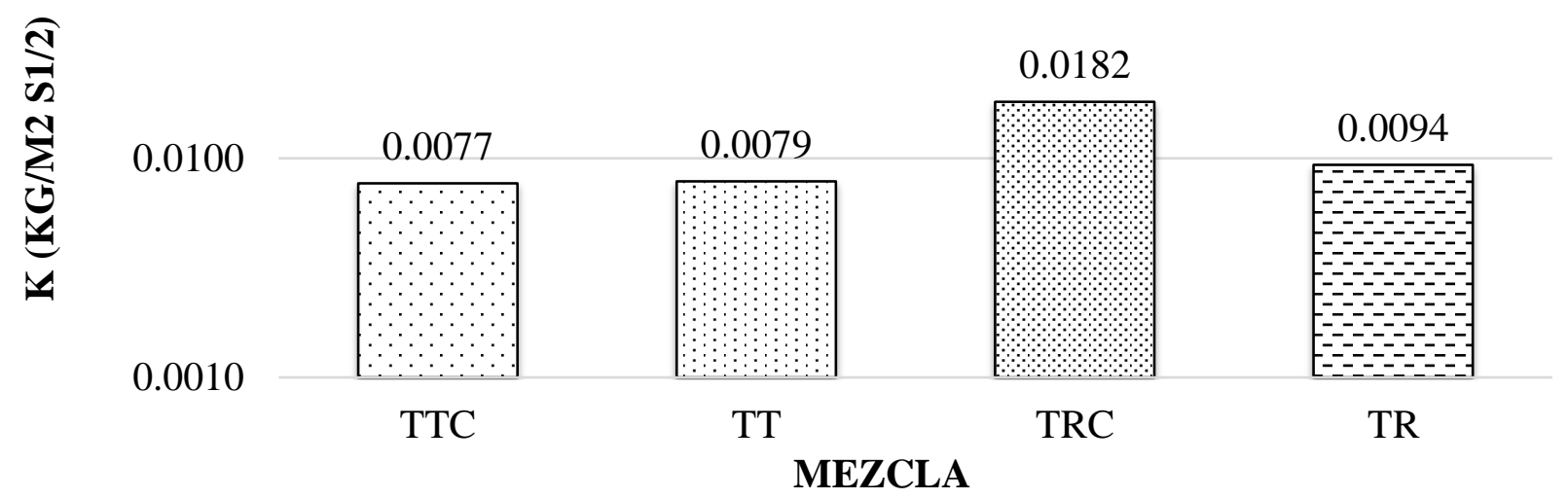

Figura 3. Absorción capilar.

En la figura 4 se presentan los resultados de la sorción capilar, los cuales demuestran que el concreto testigo triturado y el testigo redondeado presentan una mejor absorción capilar.

\section{Sorción Capilar (s)}

0.01

$\sum_{\infty}^{\widehat{S}} 0.001$

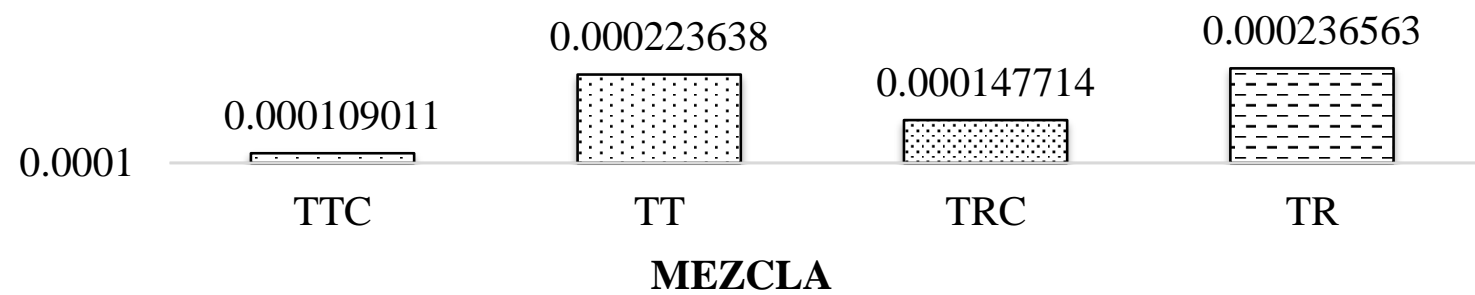

Figura 4. Sorción capilar.

\section{CONCLUSIONES}

Se observó que la incorporación de la fibra de cactus opuntia mejora la resistencia a la penetración de agua tanto para la mezcla con agregado redondeado como para la mezcla con agregado triturado, esto debido a que las fibras absorben y retienen el agua en contacto. Sin embargo, se observó en el estudio de porosidad efectiva que la fibra genera mayor cantidad de poros conectados en las mezclas, lo cual genera menor durabilidad en los elementos, tal es el caso que la muestra de agregado triturado con la 
adición de fibra presento valores superiores al 10\%, que indican un concreto de moderada calidad; lo cual queda demostrado en la gráfica de absorción capilar, ya que esta misma mezcla presenta el mayor valor respecto a las otras 3 mezclas. Por último, la gráfica de sorción capilar demuestra que todas las mezclas presentan resultados inferiores a $5 \times 10^{-5}$, lo cual indica que estos concretos son de buena calidad, sin embargo, las mezclas que no contienen adición de cactus presentan más ascensión capilar, esto debido a que no existe fibra que retenga el agua y esta transita libremente en sentido vertical. Este estudio fue de gran importancia, ya que se tenía la hipótesis de que las fibras causarían graves problemas a las estructuras, sin embargo, se demostró que benefician el fenómeno de durabilidad de los concretos.

\section{AGRADECIMIENTOS}

Los autores agradecen el financiamiento de la Coordinación de la Investigación Científica de la Universidad Michoacana de San Nicolás de Hidalgo, el soporte técnico del personal del Laboratorio de Materiales "Ing. Luis Silva Ruelas" de la Facultad de Ingeniería Civil de la Universidad Michoacana de San Nicolás de Hidalgo.

El cemento empleado en esta investigación fue proporcionado por Holcim, APASCO, por medio del Ing. Víctor Manuel Chávez del Valle.

\section{REFERENCIAS}

American Concrete Institute, Always Advancing (2002), ACI 211.1-91: Standard Practice for Selecting Proportions for Normal, Heavyweight, and Mass Concrete (Reapproved 2009). Reported by ACI Committee 211. ISBN: 9780870310171

ASTM International. (2001). C128-01 Standard Test Method for Density, Relative Density (Specific Gravity), and Absorption of Fine Aggregate. (Método de ensayo para determinar la densidad, densidad relativa (Gravedad específica) y la absorción de los agregados finos) Retrieved from https://doi.org/10.1520/C0128-01

DURAR (2000), "Manual de inspección, evaluación y diagnostico en estructuras de hormigón armado". Durabilidad de la armadura. Red temática XV.B. Programa Iberoamericano de ciencia y tecnología para el desarrollo (CYTED). Tercera edición, agosto de 2000. ISBN 980-296-541-3.

Organismo Nacional de Normalización y Certificación de la Construcción y Edificación, S.C. (2004), NMX-C-030-ONNCCE-2004: Industria de la construcción -Agregados - Muestreo. ONNCCE, S. C., México.

Organismo Nacional de Normalización y Certificación de la Construcción y Edificación, S.C. (1997), NMX-C-059-1997-ONNCCE: Industria de la construcción - determinación del tiempo de fraguado de cementantes hidráulicos (método de Vicat). ONNCCE, S. C., México.

Organismo Nacional de Normalización y Certificación de la Construcción y Edificación, S.C. (2004), NMX-C-073-ONNCCE-2004: Industria de la construcción - agregados - masa volumétrica - método de prueba. ONNCCE, S. C., México.

Organismo Nacional de Normalización y Certificación de la Construcción y Edificación, S.C. (1997), NMX-C-077-1997-ONNCCE: Industria de la construcción - agregados para concreto - análisis granulométrico - método de prueba. ONNCCE, S. C., México.

Organismo Nacional de Normalización y Certificación de la Construcción y Edificación, S.C. (2004), NMX-C-122-2004-ONNCCE: Industria de la construcción - agua para concreto - especificaciones. ONNCCE, S. C., México.

Organismo Nacional de Normalización y Certificación de la Construcción y Edificación, S.C. (2010), NMX-C-152-ONNCCE-2010: Industria de la construcción-Cementantes hidráulicos- Método de prueba Para la determinación del peso específico de cementantes hidráulicos. ONNCCE, S. C., México. 
Organismo Nacional de Normalización y Certificación de la Construcción y Edificación, S.C. (2004), NMX-C-159-2004-ONNCCE: Industria de la construcción - Concreto -Elaboración y curado, en el laboratorio de especímenes. ONNCCE, S. C., México.

Organismo Nacional de Normalización y Certificación de la Construcción y Edificación, S.C. (2004), NMX-C-164-ONNCCE-2004: Industria de la construcción - Agregados- determinación de la masa específica y Absorción de agua del agregado grueso. ONNCCE, S. C., México.

Organismo Nacional de Normalización y Certificación de la Construcción y Edificación, S.C. (2004), NMX-C-165-ONNCCE-2004: Industria de la construcción - Agregados Masa específica y absorción de agua del agregado fino - método de prueba. ONNCCE, S. C., México.

Organismo Nacional de Normalización y Certificación de la Construcción y Edificación, S.C. (2006), NMX-C-166-ONNCCE-2006: Industria de la construcción - Agregados - Contenido total de humedad por secado método de prueba. ONNCCE, S. C., México. 